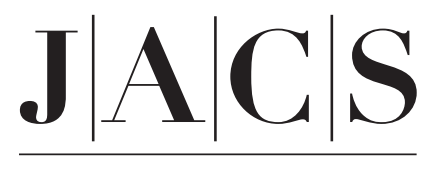

A R T I C L E S

Published on Web 05/19/2004

\title{
A Simple Approach to Sensor Discovery and Fabrication on Self-Assembled Monolayers on Glass
}

\author{
Lourdes Basabe-Desmonts, ${ }^{\dagger}$ Joris Beld, ${ }^{\dagger}$ Rebecca S. Zimmerman, ${ }^{\dagger}$ \\ Jordi Hernando,‡ Petra Mela,§ María F. García Parajo,‡ Niek F. van Hulst,‡ \\ Albert van den Berg, ${ }^{\S}$ David N. Reinhoudt, ${ }^{\dagger}$ and Mercedes Crego-Calama* ${ }^{\star}$ \\ Contribution from the Department of Supramolecular Chemistry and Technology, Applied Optics \\ Group, Department of Applied Physics, and BIOS, MESA \\ Institute for Nanotechnology, University of Twente, P.O. Box 217, \\ 7500 AE Enschede, The Netherlands \\ Received January 7, 2004; E-mail:m.cregocalama@utwente.nl
}

\begin{abstract}
Self-assembled monolayers (SAMs) on glass were used as a platform to sequentially deposit fluorophores and small molecules for ion sensing. The preorganization provided by the surface avoids the need for complex receptor design, allowing for a combinatorial approach to sensing systems based on small molecules. The resulting libraries are easily measured and show varied responses to a series of both cations and anions. This technology is transferable from the macro- to the microscale both via microcontact printing $(\mu \mathrm{CP})$, where the fluorophore is printed onto a glass surface, and via direct attachment of the fluorophore to microchannel walls. The ease of miniaturization of this technology may make the generation of a wide variety of simple yet efficient microarrays possible.
\end{abstract}

\section{Introduction}

The importance of the chemical sensing of ions and small organic molecules is illustrated across a broad range of areas encompassing medical diagnostics, environmental monitoring, and the food industry. Surface-confinement of chemical systems has facilitated the process of sensor testing. A device which allows for facile, simultaneous, multiple analyte sensing is a microsensor array. Microsensor arrays, comprised of spatially addressable ensembles of different receptors on such varied platforms as polymer supports, agarose beads, or glass slides, allow for the rapid assessment of targets with a desired catalytic or biological activity. ${ }^{1}$ This strategy has been widely used for biological purposes that necessitate rapid screening such as determining enzyme activity, ${ }^{2}$ recognizing DNA sequences, ${ }^{3}$ and characterizing monosaccharide solutions. ${ }^{4}$ Microarray technology has also been introduced for chemical sensing, as evidenced by the rapid development of electronic tongues ${ }^{5,6}$ and noses. ${ }^{7}$ Unlike biologically based arrays, which rely on the highly

$\dagger$ Department of Supramolecular Chemistry and Technology.

Applied Optics Group, Department of Applied Physics.

$\S$ BIOS.

(1) Lam, K. S.; Renil, M. Curr. Opin. Chem. Biol. 2002, 6, 353-358.

(2) Chen, G. Y. J.; Uttamchandani, M.; Zhu, Q.; Wang, G.; Yao, S. Q. ChemBioChem 2003, 4, 336-339.

(3) Steemers, F. J.; Ferguson, J. A.; Walt, D. R. Nat. Biotechnol. 2000, 18, 91-94.

(4) Curey, T. E.; Salazar, M. A.; Oliveira, P.; Javier, J.; Dennis, P. J.; Rao, P.; Shear, J. B. Anal. Biochem. 2002, 303, 42-48.

(5) Lavigne, J. L.; Savoy, S.; Clevenger, M. B.; Ritchie, J. E.; McDoniel, B.; Yoo, S.-J.; Anslyn, E. V.; McDevitt, J. T.; Shear, J. B.; Neikirk, D. J. Am. Chem. Soc. 1998, 120, 6429-6430.

(6) Di Natale, C.; Macagnano, A.; Davide, F.; D'Amico, A.; Legin, A.; Vlasov, Y.; Rudnitskaya, A.; Selezenev, B. Sens. Actuators, B 1997, 44, 423-428.

(7) Dickinson, T. A.; White, J.; Kauer, J. S.; Walt, D. R. Nature 1996, 382, $697-700$

10.1021/ja049901o CCC: $\$ 27.50$ @ 2004 American Chemical Society specific and selective interactions between substrates and targets, the few examples of chemical arrays are based on what is known as differential sensing. Here, the individual sensors are not designed to complex one unique analyte, but rather are intended to be generally responsive through nonspecific interactions to a range of analytes. ${ }^{8,9}$

The inherent strength of microarrays is enhanced by combining the technology with combinatorial chemistry. ${ }^{10}$ The use of combinatorial chemistry to generate microarrays has resulted in a broad range of DNA, ${ }^{11}$ protein, ${ }^{12}$ antibody, ${ }^{13}$ and peptide ${ }^{14}$ microarrays on surfaces such as optical fiber tips, microbeads, and microwells. ${ }^{1}$ The generation of small molecule arrays for the purpose of ion sensing, however, has thusfar been limited to polymer-immobilized dye molecules. ${ }^{15,16}$

Combining the use of combinatorial chemistry with the generation of small molecule microarrays, we have developed an effective, simple, and completely new methodology for the production of fluorescent surface sensing systems. This methodology is comprised of self-assembled monolayers (SAMs)

(8) Lavigne, J. L.; Anslyn, E. V. Angew. Chem., Int. Ed. 2001, 40, 31193130 .

(9) Birkert, O.; Tünnemann, R.; Jung, G.; Gauglitz, G. Anal. Chem. 2002, 74, $834-840$.

(10) Nicolaou, K. C., Hanko, R., Hartwig, W., Eds. Handbook of Combinatorial Chemistry; Wiley-VCH: Weinheim, GE, 2002; Vol. 2.

(11) Heller, M. J. Annu. Rev. Biomed. Eng. 2002, 4, 129-153.

(12) Kawahashi, Y.; Doi, N.; Takashima, H.; Tsuda, C.; Oishi, Y.; Oyama, R.; Yonezawa, M.; Miyamoto-Sato, E.; Yanagawa, H. Proteomics 2003, 3, $1236-1243$

(13) Nashat, A. H.; Moronne, M.; Ferrari, M. Biotechnol. Bioeng. 1998, 60, 137-146.

(14) Salisbury, C. M.; Maly, D. J.; Ellman, J. A. J. Am. Chem. Soc. 2002, 124, $14868-14870$

(15) Wu, X.; Kim, J.; Dordick, J. S. Biotechnol. Prog. 2000, 16, 513-516.

(16) Mayr, T.; Liebsch, G.; Klimant, I.; Wolfbeis, O. S. Analyst 2002, 127, $201-203$. 


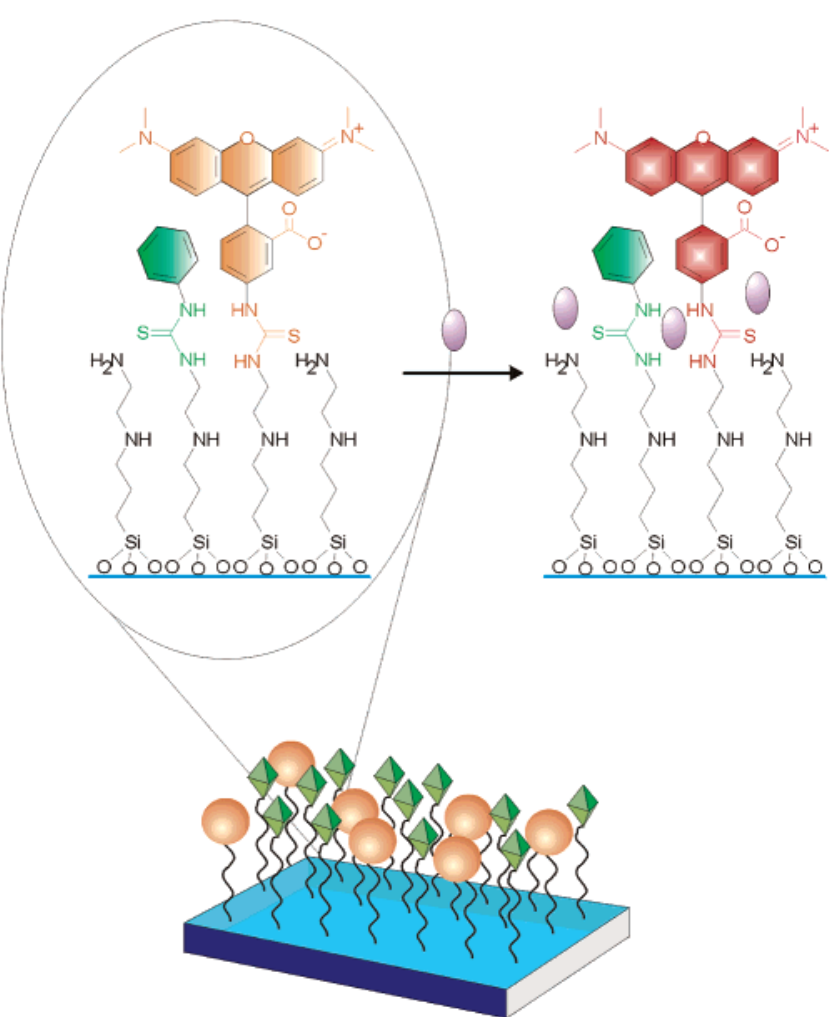

Figure 1. Schematic representation of a fluorescent self-assembled monolayer. The bottom schematic depicts binding groups by octahedrons and fluorescent groups by orange spheres. The top schematic illustrates how an analyte (purple ovals) can interact with the layer due to its coordinating properties, and the interaction is reported by the fluorophore.

with different complexing functionalities and fluorophores randomly distributed on a glass substrate. As a proof of principle, we have fabricated simple sensing systems capable of selective detection of metal cations and inorganic anions. Additionally, we have shown that the utility of such a system is fully extendable from the macro- to the microscale through microcontact printing $(\mu \mathrm{CP})$ and the use of microchannels.

SAMs have successfully been used to demonstrate that the molecular recognition process is feasible at the monolayer solution interface. ${ }^{17-19}$ The advantages of SAMs for surfaceconfined sensing are fast response times, ease and reproducibility of synthesis, ${ }^{18}$ and the introduction of additional chelating effects from the preorganization of the surface platform. ${ }^{20} \mathrm{We}$ thus use SAMs on a glass substrate as a 2D scaffold to impart sufficient molecular orientation to make it possible to separately deposit various binding functionalities (rather than the entire receptor molecule $)^{21}$ and the fluorophore on the surface to achieve a degree of analyte selectivity (Figure 1).

The phenomenon of proximal but spatially separable receptor-fluorophore communication has been recorded in solution. ${ }^{22}$ Fluorescence detection is particularly desirable due to its high sensitivity and submillisecond temporal resolution, but it has hardly ever been used to determine host-guest interactions at

(17) Kaifer, A. E. Isr. J. Chem. 1996, 36, 389-397.

(18) Crooks, R. M.; Ricco, A. J. Acc. Chem. Res. 1998, 31, 219-227.

(19) Crego-Calama, M.; Reinhoudt, D. N. Adv. Mater. 2001, 13, 1171-1174.

(20) Major, R. C.; Zhu, X.-Y. J. Am. Chem. Soc. 2003, 125, 8454-8455.

(21) van der Veen, N. J.; Flink, S.; Deij, M. A.; Egberink, R. J. M.; van Veggel, F. C. J. M.; Reinhoudt, D. N. J. Am. Chem. Soc. 2000, 122, 6112-6113.

(22) Grandini, P.; Mancin, F.; Tecilla, P.; Scrimin, P.; Tonellato, U. Angew. Chem., Int. Ed. 1999, 38, 3061-3064. the monolayer surface, mainly because most of the systems designed so far have been on gold, which causes quenching of the fluorescence. ${ }^{23,24}$ Glass, however, is an appropriate substrate for the purpose of fluorescent detection of chemical sensing. ${ }^{21}$

The directional preorganization inherent in the SAM as well as random distribution brings the binding groups and fluorophores in close enough proximity that the binding group-analyte interaction is communicated to the fluorophore, resulting in a modulation of the fluorescence intensity. In contrast to the traditional design of molecular receptors as chemical sensors based on geometric and electronic complementarity of the fluorophore-appended host to match that of the guest, our method lacks the design and the tedious synthetic efforts necessary to both label the receptor (and/or the analyte) and obtain binding selectivity.

\section{Results and Discussion}

Macroscale Cation and Anion Libraries. To explore the broad scope of this approach in regards to component variability, a quartz slide was functionalized with a SAM of $\mathrm{N}$-[3(trimethoxysilyl)propyl]ethylenediamine (TPEDA). Onto this amino-terminated monolayer were sequentially covalently attached a long excitation wavelength fluorophore (TAMRA or lissamine) and a binding group (i.e., amino, aryl-urea, alkylurea, aryl-amide, alkyl-amide) (Figures 1 and 2A). Each layer was characterized by contact angle and ellipsometry, and XPS measurements were performed on one set of layers, confirming the introduction of the components (see Supporting Information). Fluorescence measurements were taken with $10^{-4} \mathrm{M}$ acetonitrile solutions of perchlorate salts of $\mathrm{Cu}^{2+}, \mathrm{Co}^{2+}, \mathrm{Pb}^{2+}$, and $\mathrm{Ca}^{2+}$. In each instance, the layer response to the analytes occurred within seconds, and furthermore was regenerated with reproducible results upon washing with $1 \mathrm{~N} \mathrm{HCl}$. The different binding group-fluorophore combinations resulted in a range of different responses (Figure 2B).

Comparing the responses within the fluorophore series, changes in the nature of the binding group significantly affect the binding profiles. For example, both of the TAMRA layers, ureido-substituted TM1 and amido-substituted TM3, showed an increase in fluorescence upon addition of $\mathrm{Ca}^{2+}$ and $\mathrm{Pb}^{2+}$, with a greater increase for the TM1 layer than the TM3 layer. More impressive is the comparison of ureido-substituted layers TM1 and TM2. While TM1 shows increased fluorescence for both $\mathrm{Pb}^{2+}(65 \%)$ and $\mathrm{Ca}^{2+}(90 \%), \mathrm{TM} 2$, which differs by changing the isopropylphenyl substituent on the binding group for a hexyl group, shows very little change in fluorescence in the presence of these cations. The differences seen between binding groups substituted with an aryl versus an alkyl group are remarkable considering that these substituents, in principle, should not directly coordinate either $\mathrm{Pb}^{2+}$ or $\mathrm{Ca}^{2+}$, and this is a pointed example of the power of the combinatorial method. Because of the ease of generating different sensing system pairs, it requires minimal effort to synthesize layers with these small variations, which would usually be disregarded in solution-state receptor synthesis.

Within the series of lissamine layers, amino-substituted L0 and ureido-substituted L1 showed substantial increases in

(23) Motesharei, K.; Myles, D. C. J. Am. Chem. Soc. 1998, 120, 7328-7336. (24) Flink, S.; van Veggel, F. C. J. M.; Reinhoudt, D. N. Adv. Mater. 2000, 12, $1315-1328$. 

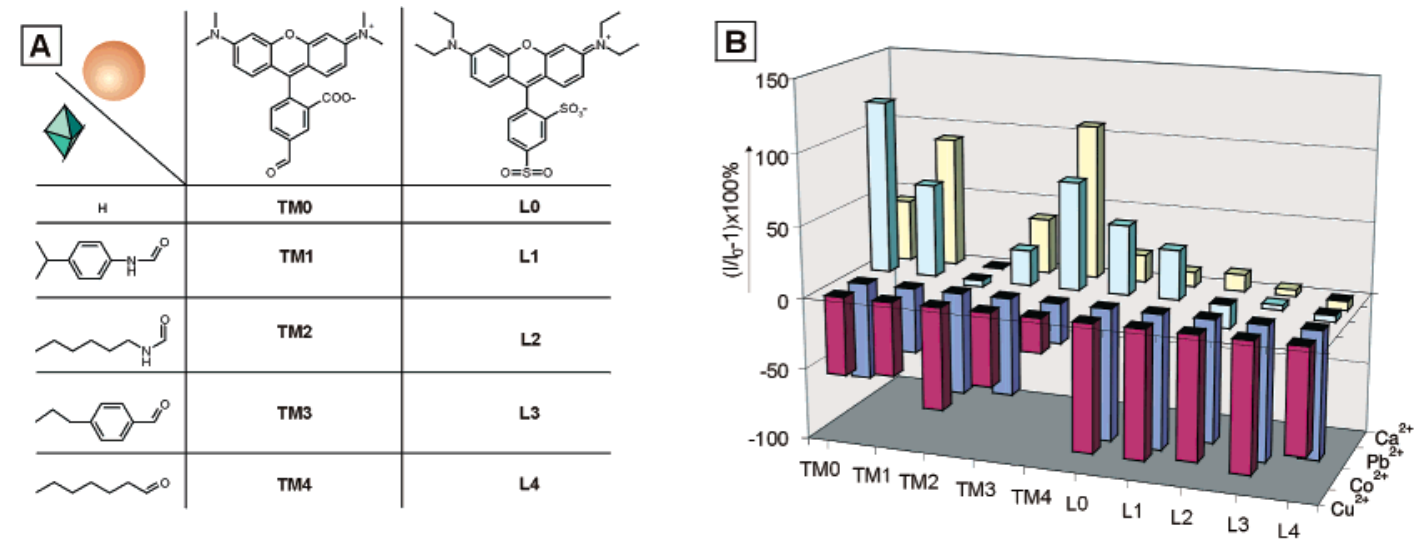

Figure 2. (A) Schematic representation for the monolayers of the cation sensing library. (B) Relative fluorescence intensity of surfaces modified with different fluorophores and chemical functionalities in the presence of $10^{-4} \mathrm{M}$ solutions of $\mathrm{Pb}^{2+}, \mathrm{Ca}^{2+}, \mathrm{Co}^{2+}$, and $\mathrm{Cu}^{2+}$ as perchlorate salts in acetonitrile. The data have been normalized; in the absence of metal cations, the fluorescence emission at 585 and $588 \mathrm{~nm}$ for layers TM0-TM4 and L0-L4, respectively, is set to 0 .

response to $\mathrm{Pb}^{2+}$, while virtually no response was seen from other amido and ureido layers L2-L4. On the other hand, systems L2-L4 show a very large fluorescence quenching upon addition of $\mathrm{Co}^{2+}$ and $\mathrm{Cu}^{2+}(\sim 80 \%)$, while no response was seen for $\mathrm{Ca}^{2+}$ and $\mathrm{Pb}^{2+}$.

Furthermore, when comparing responses between the two fluorophores, marked differences can be seen between hexyl amido TAMRA system TM4, which shows fluorescence intensity increases for both $\mathrm{Ca}^{2+}(107 \%)$ and $\mathrm{Pb}^{2+}(75 \%)$, and the corresponding hexyl amido lissamine system L4, which exhibits virtually no response for these cations. Overall, all systems exhibited a marked fluorescence intensity decrease for $\mathrm{Co}^{2+}$ and $\mathrm{Cu}^{2+}$, although more quenching was seen in the lissamine layers than in the corresponding TAMRA layers. Additionally, TM0 is the best for the sensing of $\mathrm{Pb}^{2+}(121 \%)$ versus the other ions due to the large response differences $\left(\mathrm{Ca}^{2+}\right.$ $43 \%, \mathrm{Co}^{2+}-68 \%$, and $\mathrm{Cu}^{2+}-7 \%$ ).

Several successful competition studies were performed with these layers. Here, we report as an example the studies on alkyl ureido layer L2 and alkyl amido layer L4. Addition of $10^{-4} \mathrm{M}$ $\mathrm{Ca}^{2+}$ to layer L4 induced a $10 \%$ fluorescence intensity decrease (see Supporting Information). A further addition of $10^{-4} \mathrm{M} \mathrm{Cu}^{2+}$ induced an additional $55 \%$ quenching. When reversing the addition, $10^{-4} \mathrm{M}$ of $\mathrm{Cu}^{2+}$ caused a $73 \%$ decrease in signal, and further addition of $10^{-4} \mathrm{M} \mathrm{Ca}^{2+}$ had no effect, indicating that the layer is selective for $\mathrm{Cu}^{2+}$ in the presence of $\mathrm{Ca}^{2+}$. Similar but more dramatic findings were observed for the L2 system. When $\mathrm{Ca}^{2+}$ was added, a $20 \%$ increase in signal was seen, and upon subsequent addition of $\mathrm{Cu}^{2+}, 80 \%$ quenching was observed. As was the case with L4, when the analyte addition was reversed, $\mathrm{Cu}^{2+}$ induced a $90 \%$ quenching, with no further response upon addition of $\mathrm{Ca}^{2+}$. Additional studies showed that the detection limit of these layers for the different cations is $10^{-7}-10^{-6} \mathrm{M}$ (see Supporting Information).

We thus have determined that the variables of fluorophores, binding groups, and their substituents each have a significant impact on the sensitivity and selectivity of the sensing system toward a series of cations. The binding abilities of individual fluorophore-binding group pairs are likely due to a combination of factors such as where the cation is binding, whether the binding is shared between various surface functionalities, and whether there are steric constraints or additional intermolecular surface interactions induced by noncoordinating substituents (such as changes in monolayer packing, van der Waals forces, cation $-\pi$ interactions, or $\pi-\pi$ interactions between the monolayer substituents). It is likely that some or all of these factors combine to make each pair a truly unique sensing surface. When quenching by the analyte dominates, reduction of the fluorescence is observed. Increased fluorescence, however, is a delicate interplay between quenching by both the analyte and the binding groups. If the binding groups are already quenching the fluorescence, resulting in a lowered initial fluorescence intensity, it is possible that "unquenching" can be induced upon addition of the analyte, which could lead to increased fluorescence. If the binding groups are not quenching, the fluorescence is already at a maximum and no increase can be expected; therefore, in some cases, no change in fluorescence is observed.

To further investigate the generality of this approach, we turned toward the historically more difficult field of anion sensing. Anion sensing development in terms of both sensitivity and selectivity lags behind that of cations across the entire range of sensing systems, from solution state to CHEMFETs and ion selective electrodes. ${ }^{25}$ The reasons for this include such factors as their larger size-to-charge ratio, large heat of hydrations, and geometric constraints. ${ }^{26}$

An amino SAM on a quartz slide was functionalized sequentially, in the manner previously described, with one fluorophore (TRITC or lissamine) and one known anion binding functional group (i.e., amino, amide, sulfonamide, urea, or thiourea) (Figure 3A). These layers were characterized by contact angle and ellipsometry measurements (see Supporting Information), and the fluorescence response of the layers to $10^{-4}$ $\mathrm{M}$ acetonitrile solutions of tetrabutylammonium salts of $\mathrm{HSO}_{4}{ }^{-}$, $\mathrm{NO}_{3}{ }^{-}, \mathrm{H}_{2} \mathrm{PO}_{4}^{-}$, and $\mathrm{AcO}^{-}$anions was recorded (Figure $3 \mathrm{~B}$ ). As in the case of sensing the cations, each layer revealed a different response pattern to the anions.

As a general trend, the TRITC systems T0-T4 exhibited a larger magnitude of response to all of the anions than did the corresponding lissamine systems L0, L5-L8, which illustrates the differences between the two fluorophores. This difference is likely due to the attachment point functionality. TRITC forms a thiourea bond when reacted with the layer, while lissamine

(25) Antonisse, M. M. G.; Snellink-Ruël, B. H. M.; Lugtenberg, R. J. W Engbersen, J. F. J.; van den Berg, A.; Reinhoudt, D. N. Anal. Chem. 2000 $72,343-348$

(26) Beer, P. D.; Gale, P. A. Angew. Chem., Int. Ed. 2001, 40, 486-516. 

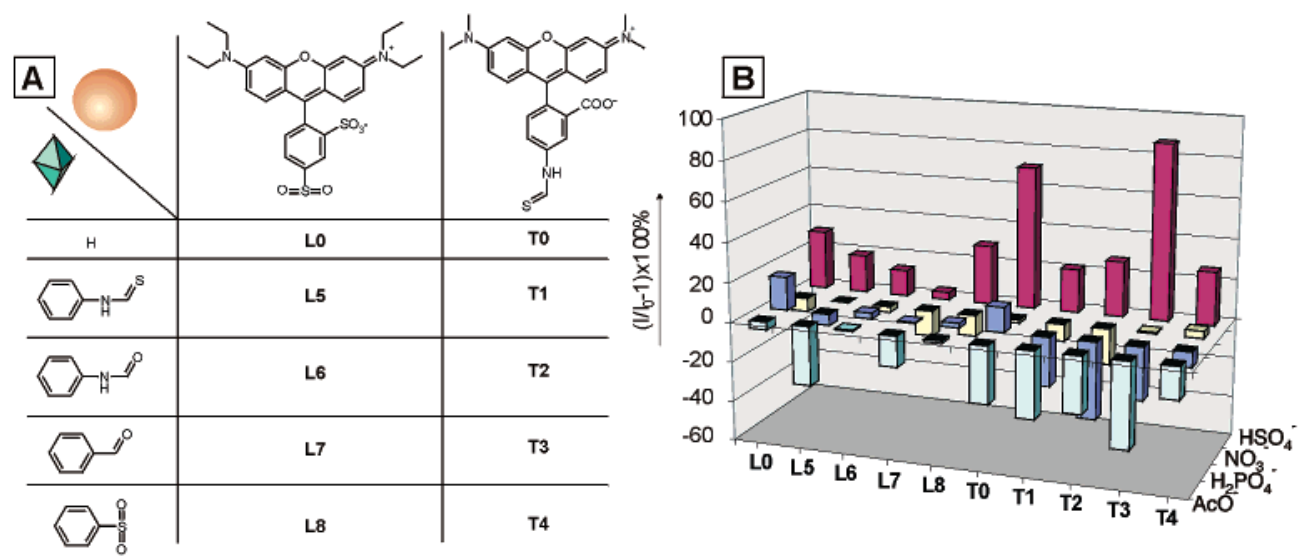

Figure 3. (A) Schematic representation for the monolayers of the anion sensing library. (B) Relative fluorescence intensity of surfaces modified with different fluorophores and chemical functionalities in the presence of $10^{-4} \mathrm{M}$ solutions of $\mathrm{HSO}_{4}^{-}, \mathrm{NO}_{3}{ }^{-}, \mathrm{H}_{2} \mathrm{PO}_{4}^{-}$, and $\mathrm{AcO}^{-}$as tetrabutylammonium salts in acetonitrile. The data have been normalized; in the absence of anions, the fluorescence emission at 585 and $575 \mathrm{~nm}$ for layers L0, L5-L8, and T0-T4, respectively, is set to 0

forms a sulfonamide bond. An especially noteworthy response is the large sensitivity of layers $\mathrm{T} 1, \mathrm{~T} 2$, and $\mathrm{T} 3$ toward $\mathrm{H}_{2} \mathrm{PO}_{4}{ }^{-}$, showing fluorescence quenching from $35 \%$ to $50 \%$.

Within the lissamine series, L5, functionalized with a thioureido group, suffers a quenching of $35 \%$ in the presence of $\mathrm{AcO}^{-}$, while the same layer has a moderate increase of fluorescence in the presence of $\mathrm{HSO}_{4}^{-}(20 \%)$.

Noteworthy and important for sensing purposes is the response of the TRITC-functionalized layers T1-T3. The fluorescence response of these layers increased between $24 \%$ and $87 \%$ in the presence of $\mathrm{HSO}_{4}{ }^{-}$, while the same layers showed a fluorescence intensity decrease in the presence of $\mathrm{H}_{2} \mathrm{PO}_{4}^{-}$between $35 \%$ and $56 \%$.

These varied responses (especially the increases in fluorescence) across the library help decrease the chances of false positives for the individual analytes. Additionally, aminofunctionalized $\mathrm{T} 0$ is an excellent sensor for $\mathrm{HSO}_{4}^{-}$; not only is the magnitude of fluorescence increase quite large (72\%), but it is the only anion that induces such an increase. The addition of $\mathrm{AcO}^{-}$induces a quenching of $34 \%$, while $\mathrm{NO}_{3}{ }^{-}$and $\mathrm{H}_{2} \mathrm{PO}_{4}{ }^{-}$ result in virtually no response. These results support those found for the previous cation systems, wherein making a "library" of fluorophores and binding groups results in a unique array of responses to the anions.

Additional studies showed that even at anion concentrations as low as $10^{-5} \mathrm{M}$, systems T0-T4, L0, and L5 already exhibit sensitivity toward both $\mathrm{AcO}^{-}$and $\mathrm{HSO}_{4}^{-}$(see Supporting Information). Furthermore, two selectivity experiments for the binding of $\mathrm{HSO}_{4}{ }^{-}, \mathrm{NO}_{3}{ }^{-}$, and $\mathrm{AcO}^{-}$were also conducted with T3 layers (see Supporting Information). In both experiments, first $10^{-4} \mathrm{M}$ of $\mathrm{NO}_{3}{ }^{-}$was added to a T3 layer, showing no response as was expected. Next, two separate experiments were performed reversing subsequent additions of solutions of $\mathrm{HSO}_{4}{ }^{-}$ and $\mathrm{AcO}^{-}$, ranging from $10^{-6}$ to $10^{-3} \mathrm{M}$. In each case, the addition of $\mathrm{AcO}^{-}$caused $70 \%$ fluorescence quenching, but only when the $\mathrm{HSO}_{4}{ }^{-}$was added first did the $\mathrm{HSO}_{4}{ }^{-}$result in a fluorescence intensity increase. Thus, it appears that the system $\mathrm{T} 3$ is able to selectively sense $10^{-5} \mathrm{M} \mathrm{AcO}^{-}$in the presence of $10^{-4} \mathrm{M} \mathrm{NO}_{3}^{-}$and $10^{-3} \mathrm{M} \mathrm{HSO}_{4}^{-}$.

These findings could represent an important step forward in the field of anion binding, which often requires significant synthetic effort to achieve binding sensitivity and selectivity.
Indeed, the general implications of these initial findings toward the detection of both cations and anions are significant across the entire field of chemical analyte sensing. Through a combinatorial approach to the generation of chemical sensors from commercially available, simple small molecules, and by means of a simple analytical protocol, we have the ability to generate in situ a huge number of sensing surfaces without the necessity of target labeling or library deconvolution to determine successful sensing systems. The overall simplicity of the system is its strength, in that it imparts generality to the methodology in terms of both sensing system generation and the exploration of microanalysis technology. Additionally, it can be envisioned that, given a data set containing a number of general trends, a series when taken as a whole "fingerprint" represents a unique analyte response.

We believe that the method is particularly amenable to the transition toward miniaturization and subsequent array format, which would open the possibility for a large number of sensing systems to be quickly synthesized and screened. This would allow both for fingerprints to be made to discern the lead sensing architectures for the detection of analyte mixtures, as well as for hand-picking of particularly selective combinations for a specific analyte. ${ }^{27} \mathrm{We}$ therefore desired to miniaturize the process and use fluorescence confocal microscopy to image layer response, which would allow for the accessibility of true microarrays and high throughput screening.

Microcontact Printing of the Cation Library. A possible means is through the use of microcontact printing $(\mu \mathrm{CP}),{ }^{28}$ which has been used in the past decade to successfully pattern onto surfaces of gold, quartz, and silicon. The printing of proteins, peptides, and enzymes has been extensively developed, but, surprisingly, the area of small molecule printing remains virtually unexplored. Furthermore and to the best of our knowledge, there are only three examples of covalent modification of SAMS through $\mu \mathrm{CP} .{ }^{29-31}$ Herein, we demonstrate not only the first covalent printing of sensing reporters on a reactive

(27) Phizicky, E.; Bastiaens, P. I. H.; Zhu, H.; Snyder, M.; Fields, S. Nature 2003, 422, 208-215

(28) Kumar, A.; Whitesides, G. M. Appl. Phys. Lett. 1993, 63, 2002-2004

(29) Lahiri, J.; Ostuni, E.; Whitesides, G. M. Langmuir 1999, 15, 2055-2060.

(30) Andersson, H.; Jönsson, C.; Moberg, C.; Stemme, G. Sens. Actuators, B 2001, 79, 78-84

(31) Yan, L.; Zhao, X.-M.; Whitesides, G. M. J. Am. Chem. Soc. 1998, 120, $6179-6180$. 


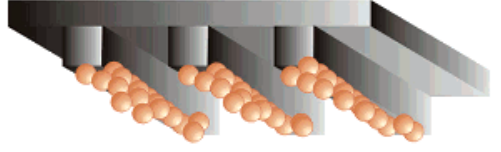

A
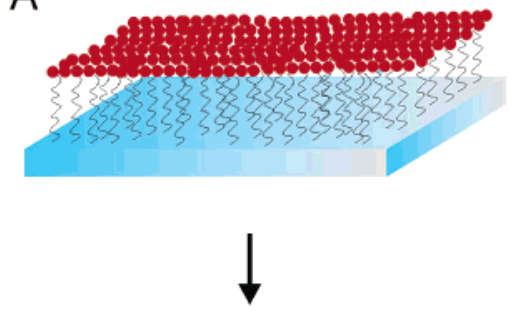

B
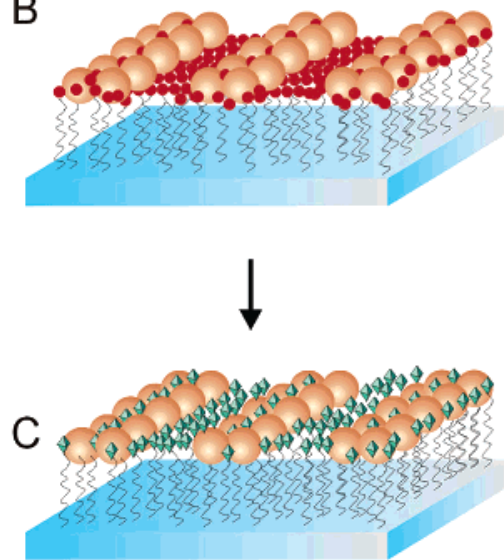

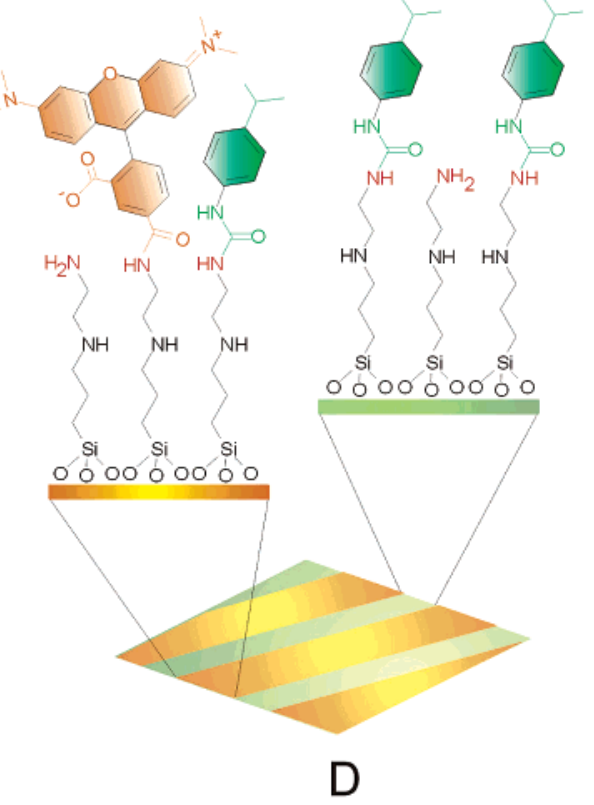

Figure 4. Generation of the patterned, sensitive monolayers using $\mu \mathrm{CP}$. (A) An amino-terminated monolayer on a glass surface is brought into contact with a PDMS stamp inked with a fluorophore. (B) This results in the covalent attachment of the fluorophore to the amino-terminated monolayer, yielding a patterned fluorescent monolayer. (C) Subsequent immersion of the sample into a solution of reactive molecules allows the functionalization of the remaining free amino groups. (D) The result of the process is a glass slide patterned with two different areas, from which one area (where the fluorophore has been printed and the reactive molecules have been attached) is sensitive to cations.

SAM on glass, but the use of $\mu \mathrm{CP}$ as a delivery tool and not simply as a patterning technique, to achieve the first microcontact printed surface for the purpose of chemical ion sensing.

An amino-functionalized TPEDA SAM was synthesized onto a glass surface as described previously. A PDMS (poly(dimethoxysiloxane)) stamp was first inked in an acetonitrile solution of the fluorophore (TAMRA or lissamine) and brought into contact with the SAM. The fluorophore is thus covalently attached to the layer, resulting in a patterned fluorescent monolayer of $5 \mu \mathrm{m}$ wide parallel lines separated by $3 \mu \mathrm{m}$. After vigorous rinsing, ${ }^{32}$ the slide was subsequently immersed in an acetonitrile solution of a reactive molecule, resulting in the attachment of binding groups onto the surface (i.e., urea or amide) at the sites of the unreacted surface amino groups (Figure 4).

The slide was first imaged in acetonitrile by laser confocal microscopy. Next, using a custom built liquid cell, the slides were immersed in a $10^{-4} \mathrm{M}$ acetonitrile solution of perchlorate salts of $\mathrm{Co}^{2+}, \mathrm{Cu}^{2+}, \mathrm{Ca}^{2+}$, or $\mathrm{Pb}^{2+}$ and imaged for fluorescence changes (Figure 5). For example, layer L3, microcontact printed with the lissamine fluorophore and reacted with $p$-propylbenzoyl chloride to form an amido binding group, resulted in quenching of $70 \%$ for $\mathrm{Cu}^{2+}$ (Figure 5A) and $82 \%$ and for $\mathrm{Co}^{2+}$, respectively, and little response toward $\mathrm{Pb}^{2+}$ and $\mathrm{Ca}^{2+}$ (Figure 5C).

(32) A control experiment was performed to probe the covalent attachment of the fluorophore to the substrate: a nonreactive monolayer prepared with decyl trichlorosilane (DTS) was fabricated following the same procedure as for TPEDA. After the fluorophore was printed, the standard rinsing protocol (see Supporting Information) was performed, resulting in the total loss of fluorescence.
On the other hand, for the TAMRA layer TM1 with a ureido binding group, responses were completely different. A dramatic $179 \%$ increase was seen in the fluorescence for $\mathrm{Ca}^{2+}$ (Figure $5 \mathrm{~A}$ and $\mathrm{B}$ ) and a $98 \%$ increase for $\mathrm{Pb}^{2+}$, while there was very little response for both $\mathrm{Co}^{2+}$ and $\mathrm{Cu}^{2+}$ (Figure 5C).

Although a quantitative comparison between two different techniques is not possible, qualitatively these results correlate well with what was previously seen for the same layers in the macroscale cation library, indicating that it is possible to transfer the methodology concept from the macro- to the microscale. In this way, it would be possible to generate a microsensing array comprised of different printed fluorophores and either cross printed with reactive molecules to obtain certain surface binding groups, or reacted with them in solution. The system has a builtin transducer allowing the direct visualization of the sensing process in conjunction with a detection method compatible with high throughput screening techniques without further labeling steps. This could lead to the fast generation of numerous sensing combinations that are easily and quickly screened for their binding abilities.

Cation Sensing in a Microchannel. We have also extended the scope of the miniaturization process by integrating the sensing system into a microchannel. A series of such individually accessible channels eliminates problems such as solvent evaporation in the preparation of the monolayer and in the analysis, as well as pollution or contamination. Additionally, each channel can be exposed to a unique analyte environment, and continuous flowing of the analyte through the channels 

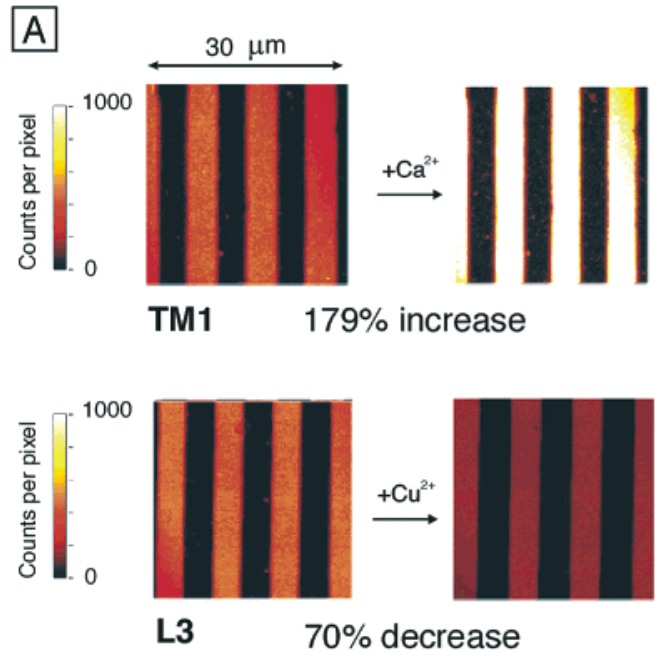

B

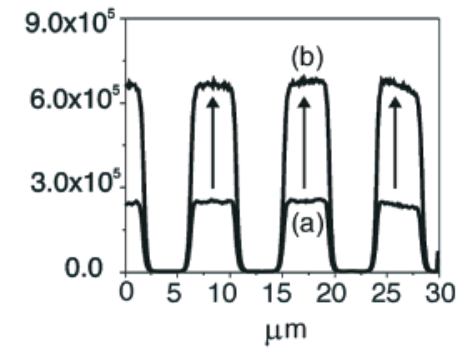

L3

$70 \%$ decrease

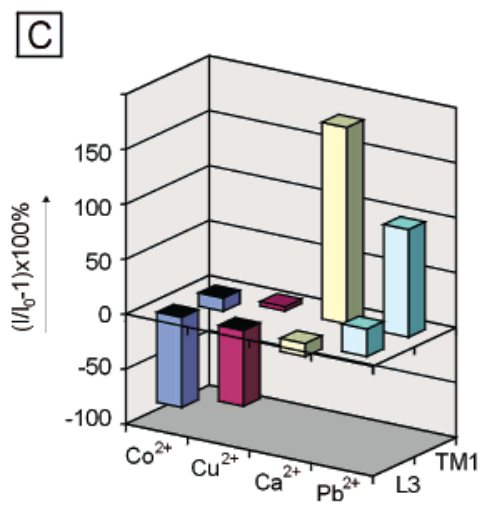

D

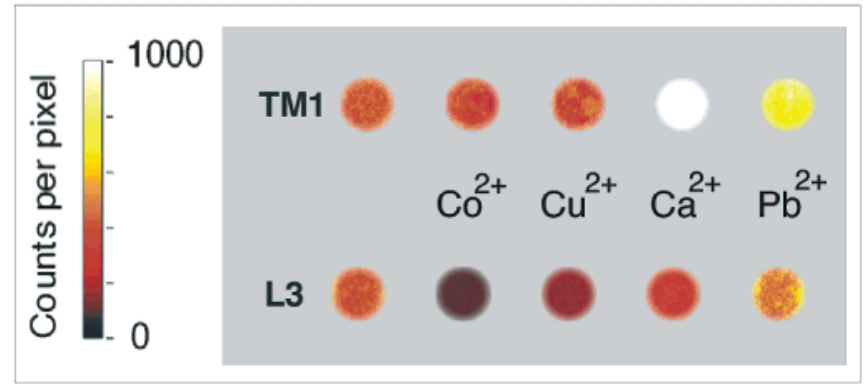

Figure 5. (A) Fluorescence confocal microscope images of the systems TM1 and L3 in contact with acetonitrile (left) and in contact with a $10^{-4} \mathrm{M} \mathrm{Ca}^{2+}$ and $\mathrm{Cu}^{2+}$ solution in acetonitrile, respectively (right). (B) Profile of cross sections of the image of TM1 before (a) and after (b) addition of Ca ${ }^{2+}$; the $y$-axis is fluorescence intensity (counts per pixel), and the $x$-axis is in micrometers. (C) Relative fluorescence intensity of systems TM1 and L3 in the presence of $10^{-4} \mathrm{M}$ acetonitrile solutions of $\mathrm{Pb}^{2+}, \mathrm{Cu}^{2+}, \mathrm{Co}^{2+}$, and $\mathrm{Ca}^{2+}$ as perchlorate salts. (D) Array of fluorescence confocal microscope images of the systems L3 and TM1 in contact with acetonitrile (first spot) and the metal salt solutions. ${ }^{33}$

allows for real-time response measurements. These advantages, in addition to its addressable platform, lend it to high throughput screening techniques and offer the opportunity to form microarrays. Microchannels are particularly attractive for our purposes because they provide a convenient platform for rapid analysis and detection as has been shown for biological samples, ${ }^{34}$ and, more recently, for chemical analytes. ${ }^{35-37}$

Until now, there has not been an example of a sensitive system covalently bound to the channel wall itself. Nevertheless, it has been shown that the glass surface of a microchannel behaves similarly to macroscale glass surfaces and is easily activated and modified with SAMs. ${ }^{38}$

(33) The printed surface is not perfectly homogeneous, and the array spots depict only a very small area and are meant to be a pictorial representation only, whereas the quantitative values for the fluorescence intensity changes are taken as an average over the whole measuring surface.

(34) Andersson, H.; van den Berg, A. Sens. Actuators, B 2003, 3, 315-325.

(35) Sato, K.; Hibara, A.; Tokeshi, M.; Hisamoto, H.; Kitamori, T. Adv. Drug Delivery Rev. 2003, 55, 379-391.

(36) Rudzinski, C. M.; Young, A. M.; Nocera, D. G. J. Am. Chem. Soc. 2002, 124, 1723-1727.

(37) Guijt, R. M.; Baltussen, E.; van der Steen, G.; Schasfoort, R. B. M.; Schlautmann, S.; Billiet, H. A. H.; Frank, J.; van Dedem, G. W. K.; van den Berg, A. Electrophoresis 2001, 22, 235-241.

(38) Munro, N. J.; Hühmer, A. F. R.; Landers, J. P. Anal. Chem. 2001, 73, 1784-1794.

(39) The synthetic protocol of flowing the monolayer components through the channel intrinsically results in the coating of the entire microchannel. Additionally, having three inlets available allowed us to fill them all with the same solution simultaneously, thus preventing evaporation of the small solvent volumes used.
To explore this possibility, a three-inlet glass microchannel of $20 \mu \mathrm{m}$ width, $12 \mathrm{~mm}$ length, and $2 \mu \mathrm{m}$ depth was hydroxylated with piranha, thoroughly rinsed with MillliQ water, and then functionalized with a toluene solution of $\mathrm{N}$-[3(trimethoxysilyl)propyl]ethylenediamine. An acetonitrile solution of TAMRA fluorophore and chloroform solution of $p$-isopropylphenyl isocyanate were sequentially flowed through the channel to form a TM1 layer (Figure 6A). Using confocal microscopy, the acetonitrile-filled channel was first imaged in the absence of analyte. A $10^{-4} \mathrm{M}$ acetonitrile solution of the perchlorate salt of $\mathrm{Pb}^{2+}$ was then flowed through, resulting in a 50\% increase in fluorescence intensity (Figure 6B and C). Afterward, the channel was rinsed sequentially with $\mathrm{HCl}(0.1$ $\mathrm{N}), \mathrm{NaHCO}_{3}(5 \%)$, MilliQ $\mathrm{H}_{2} \mathrm{O}$, EtOH, and $\mathrm{CH}_{2} \mathrm{Cl}_{2}$, and then dried in a stream of air. Reproducible changes in the fluorescence intensities were then obtained from a subsequent addition of the $\mathrm{Pb}^{2+}$ solution. Thus, we were able to generate the first chemical sensing, microchannel device with the sensing system incorporated into the channel walls.

The power of this method is such that in a network with different fluorophore-binding group mixtures in each channel, it will be possible to generate a fingerprint of the entire network

(40) The confocal microscopy measurements were imaged in the $x y$ plane. The intensity of the laser was focused on only one wall of the microchannel, resulting in higher intensity on the edges. 


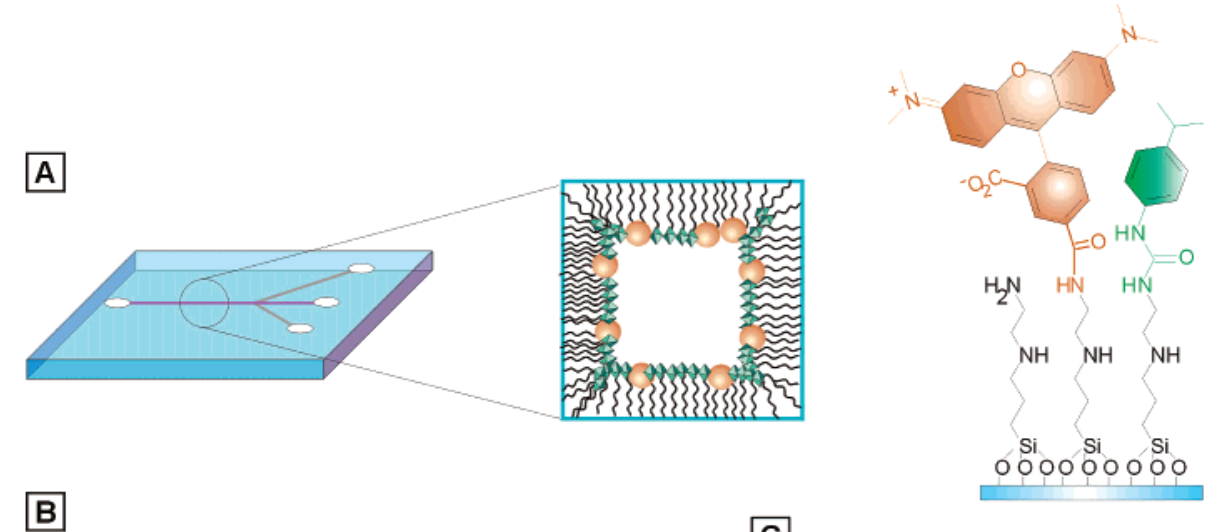

B

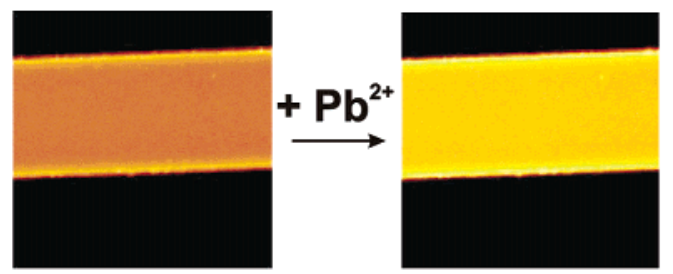

C

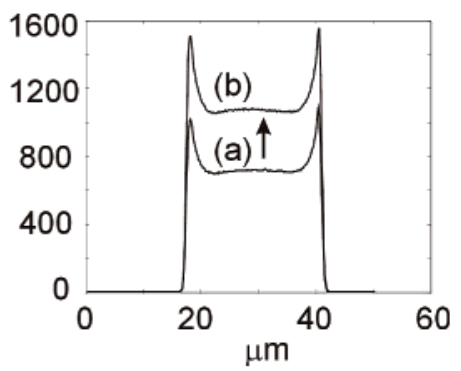

Figure 6. (A) Schematic representation and enlarged cross section of the microchannel funtionalized with the system TM1.39 (B) $70 \times 70 \mu \mathrm{m}$ confocal microscopy images of the channel: ${ }^{40}$ filled with acetonitrile (left) and filled with a $10^{-4} \mathrm{M}$ solution of $\mathrm{Pb}^{2+}$ in acetonitrile (right). The image reveals an increase of $50 \%$ in the fluorescence intensity. (C) Fluorescence intensity profiles of the images: (a) in acetonitrile; (b) in $10^{-4} \mathrm{M}$ solution of $\mathrm{Pb}^{2+}$ in acetonitrile. The $y$-axis is fluorescence intensity (counts per pixel).

with a single fluorescence "snapshot" for a large number of analytes. By combining these snapshots of the array for each analyte, it is possible to envision an easily generated "photo album" for a wide range of analytes for any particular array. Additionally, the possibility of regenerating the channel activity will allow for the sequential testing of multiple analytes.

\section{Concluding Remarks}

Starting with the idea of building an easily generated sensing system by the combinatorial approach, we have shown that sequential deposition of a fluorophore and a binding group onto quartz results in a complete sensing system for cations and anions with no need to independently design and synthesize receptors. We have also shown that this concept is fully transferable to the microscale, as demonstrated through a new use of $\mu \mathrm{CP}$, where we generated the first covalently printed fluorophore and hence the first microcontact printed sensor on glass. In addition, we were able to produce the first covalently bound integrated sensing system in a glass microchannel, which opens the possibility of generating high throughput screening arrays.

In summary, we have developed a new and simple approach to the generation of sensing systems through the combined advantages imparted by such factors as: (a) a combinatorial approach to the generation of sensing surfaces, (b) label-free analytes and binding groups, (c) no need for analyte deconvolution to determine receptor "hits", (d) no need for a complicated analytical interface, and (e) the potential for high throughput screening. The scope of our method is such that many possibilities can be envisioned between combinations of fluorophores and any other binding group, reactive molecule, biomolecule, or specific receptor with a compatible functionality for attachment to the surface. We are currently exploring the utility of the approach with various selective complexing motifs, expanding the range of potential analytes able to be differentially sensed, and utilizing the methodology for sensor device generation to make microarrays.

Acknowledgment. The research of M.C.-C. has been made possible by a fellowship of the Royal Netherlands Academy of Arts and Sciences. J.H. thanks the European Community Program IHP-99 for a Marie Curie Fellowship (HPMF-CT2002-01698).

Supporting Information Available: Detailed experimental information, characterization data, and error analysis. This material is available free of charge via the Internet at http://pubs.acs.org.

JA0499010 